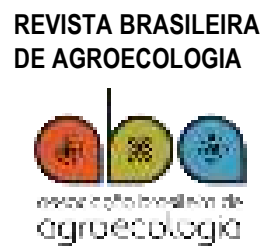

ISSN: 1980-9735

\title{
O PERFIL DE CONSUMO E PERCEPÇÃO EM RELAÇÃO AOS ALIMENTOS ORGÂNICOS DOS CONSUMIDORES GAÚCHOS
}

\author{
Consumption profile and perception in relation to organic food
}

by gaucho consumers

1 Universidade do Vale do Taquari - Univates.

${ }^{2}$ E-mail: fernanda@univates.br

${ }^{3}$ E-mail: afeil@univates.br

${ }_{4}^{4}$ E-mail: cyrne@univates.br

5 E-mail: jbarden@univates.br

6 E-mail:

marlon.dalmoro@univates.br

Recebido em:

11/09/2018

Aceito para publicação em: 06/11/2018

Correspondência para: fernanda@univates.br
Fernanda C. W. Sindelar ${ }^{1,2}$, Alexandre A. Feil ${ }^{1.3}$, Carlos C. S. Cyrne $^{1,4}$,
Júlia E. Barden ${ }^{1,5}$ e Marlon Dalmoro

RESUMO

Apesar do consumo de alimentos orgânicos ter sido alvo de inúmeros estudos, resultados contraditórios indicam que a compreensão desse fenômeno passa por análises específicas em diferentes contextos. Este trabalho tem como objetivo analisar o perfil de consumo e as percepções em relação aos alimentos orgânicos junto aos consumidores gaúchos. Adotou-se uma abordagem quantitativa por meio de levantamento junto a 2.691 consumidores de diferentes regiões do estado do Rio Grande do Sul. Os resultados apontam que os principais produtos orgânicos consumidos são vegetais, hortaliças e frutas. As razões de consumo estão associadas à busca por alimento saudável e natural, à preservação da saúde e do meio ambiente. Ressalta-se que, o consumo de orgânicos pelos gaúchos envolve a percepção de ganhos de saúde pelo fato de serem alimentos livres de pesticidas e fertilizantes. Os resultados fornecem, por meio de uma perspectiva localizada, um panorama capaz de orientar políticas públicas e estratégias comerciais de fomento ao consumo de alimentos orgânicos.

Palavras-chave: Produtos Orgânicos. Perfil de Consumo. Percepções. Rio Grande do Sul.

\section{ABSTRACT}

Although organic food consumption has been the subject of several studies, their contradictory results indicate the comprehension of this phenomenon involves the particular analysis in different contexts. This study aims to analyze the consumption profile and perceptions about organic food by consumers in the south of Brazilian state of Rio Grande do Sul. It was adopted a quantitative approach running a survey with 2.691 consumers. Results indicate that organic food consumption involves mainly vegetables, greenery and fruits. Consumption reasons are associated with a search for healthier and natural food, as well as health gains and environment preservation. We emphasize that organic food consumption in Rio Grande do Sul involves the perception of health benefits in eating food free of pesticides and fertilizers. Conclusions supply, through a localized perspective, an overview capable of orienting public policies and commercial strategies to promote the consumption of organic foods in the region.

Keywords: Organic Products. Consumption Profile. Perceptions. Rio Grande do Sul. 
Introdução

O mercado de orgânicos, em 2017, movimentou no país R\$ 3,5 bilhões, com um crescimento de cerca de $20 \%$ em relação ao ano anterior (GLOBORURAL, 2018). Apesar da agricultura convencional ser hegemônica no volume de alimento produzido no Brasil, o crescimento da agricultura orgânica tem sido acompanhado por uma exaltação a modos de produção mais sustentáveis. A agricultura orgânica se distingue da produção convencional por não envolver fertilizantes sintéticos e pesticidas, bem como por utilizar conhecimento técnico na busca por alimentos mais sustentáveis do ponto de vista social, econômico e ambiental (IFOAM, 2017).

Diante desse fenômeno, diferentes estudos têm buscado compreender as particularidades dos alimentos orgânicos nas suas mais diferentes faces. Enquanto uma série de estudos busca analisar aspectos relacionados ao produtor de alimentos orgânicos (BURTON, 2004; SUTHERLAND e DARNHOFER, 2012; PRESS et al., 2014), outra linha de estudo busca dar conta de compreender as diferentes faces do consumo deste tipo de alimentos, destacando especificidades culturais (THOMPSON e COSKUNER-BALLI, 2007; DALMORO, 2015; NUTTAVUTHISIT e THØGERSEN, 2017; entre outros), psicossociais (AERTSENS et al., 2009; THOGERSEN et al., 2015; entre outros) e de comportamento de consumo (DETTMANN e DIMITRI, 2009; GRUBOR e DJOKIC, 2016). Apesar do grande número de estudos sobre o tema, ao analisar a literatura de forma transversal, é possível identificar discrepâncias na descrição desse fenômeno do ponto de vista do consumo (SULTAN et al., 2018). Por exemplo, enquanto que os estudos iniciais, ainda no final da década de 1980, encontravam na preocupação ambiental e na busca por produtos ambientalmente corretos, a principal razão para o consumo de alimentos orgânicos (JOLLY et al., 1989), estudos recentes indicam uma preocupação com a saúde como principal razão (GRUBOR e DJOKIC, 2016; DALMORO, 2015). Assim, mesmo sendo alvo de inúmeros estudos, compreender as diferentes especificidades que envolvem o consumo de orgânicos continua sendo um desafio para os pesquisadores.

Porém, na descrição do consumidor de orgânicos contemporâneo, o que demonstra apresentar maiores discrepâncias entre um estudo e outro é a definição do perfil de consumo desse tipo de alimento (DIMITRI e DETTMANN, 2012). Como exemplo, enquanto a literatura nos Estados Unidos tem identificado distinções em termos de renda e origem étnica entre quem consome ou não alimentos orgânicos (DETTMANN e DIMITRI, 2009), na Europa pesquisadores têm identificado distinções em termos de idade e gênero (MONIER et al., 2009; JANSSEN et al., 2009; GRUBOR e DJOKIC, 2016). A explicação para isso reside no fato de que cada país ou região pode ter particularidades que auxiliam na orientação de diferentes perfis de consumidores a procurarem alimentos orgânicos. Além disso, diferentes níveis de acesso aos alimentos orgânicos podem influenciar o perfil de consumo e as percepções de quem procura esse tipo de alimento (DIMITRI e DETTMANN, 2012). Outra explicação é a incapacidade dos estudos em capturar particularidades regionais que orientam as escolhas dos consumidores. Aspectos como características socioeconômicas de cada região e hábitos alimentares específicos podem ditar o perfil de consumo de orgânicos.

Dessa forma, a realização de estudos localizados, capazes de descrever o perfil de consumo de alimentos orgânicos em diferentes regiões se faz necessária. Isso é mais relevante em países como o Brasil, dadas as suas dimensões continentais e distinções socioculturais em suas diferentes regiões. Apesar do amplo leque de estudos que analisam o perfil de consumo de orgânicos existentes no Brasil, eles se concentram no estudo de determinadas cidades (ver quadro 2) e não de regiões. Além disso, é importante ressaltar que o contexto da alimentação orgânica no Brasil carece de dados científicos, tanto no âmbito da produção quanto do consumo.

Com base no exposto, esse estudo objetiva analisar o perfil de consumo e as percepções em relação aos alimentos orgânicos junto aos consumidores gaúchos. O foco no estado do Rio Grande do Sul permite por luz em uma das principais regiões consumidoras de alimentos orgânicos no Brasil (ORGANIS, 2017). Com base nesse objetivo, os resultados contribuem para reduzir a carência de informações sobre o perfil e as escolhas dos consumidores de alimentos orgânicos. Os dados gerados, além de permitirem reconhecer se os alimentos orgânicos preservam uma distinção em relação aos convencionais, servem de suporte para orientar produtores e gestores públicos no desenvolvimento desse tipo de produção. 
Nas partes que seguem essa introdução, primeiramente é apresentada uma discussão teórica sobre o tema. Após, detalham-se os procedimentos metodológicos e os resultados obtidos no plano empírico. Por fim, são apresentadas as conclusões do estudo.

\section{Referencial teórico}

\section{A construção do orgânico como uma categoria de consumo}

A agricultura orgânica pode ser caracterizada, do ponto de vista prático, como um sistema produtivo que visa sustentar a saúde dos solos, dos ecossistemas e das pessoas, a partir de processos ecológicos, biodiversidades e ciclos adaptados às condições locais. De acordo com a legislação brasileira, o sistema orgânico de produção agropecuária consiste num produto orgânico in natura ou processado "[...] obtido em sistema orgânico de produção agropecuário ou oriundo de processo extrativista sustentável e não prejudicial ao ecossistema local” (BRASIL, 2003, p. 1).

Entre os princípios que fundamentam essa atividade estão (IFOAM, 2017): a) saúde: tanto dos ecossistemas como dos organismos; b) ecologia: devendo respeitar os ciclos e balanços ecológicos da natureza; c) justiça: visando a equidade e a qualidade de vida dos envolvidos; e d) cuidado: devendo ser desenvolvida de maneira preventiva e responsável para atender as gerações atuais e futuras e do meio ambiente. Esses princípios estão calcados na agroecologia, compreendida como a ciência da agricultura sustentável (ALTIERI, 2018). A agroecologia envolve uma posição filosófica e prática que visa reconectar o homem à natureza, especialmente frente às vulnerabilidades dos modelos convencionais de produção agroalimentar. Seguindo os princípios da agroecologia, a produção de alimentos deve ser guiada por modos de produção autossuficientes e sustentáveis, a partir da coexistência com o sistema natural, evitando o uso de produtos químicos, minimizando a poluição e os danos ambientais (ALTIERI, 2018).

Apesar das noções da agroecologia e sua distinção em relação à agricultura convencional estarem difundidas entre os produtores agroecologistas, do ponto de vista do consumo, os produtos oriundos da agroecologia têm sido rotulados como 'orgânicos' (MIGLIORINI e WEZEL, 2017). Esse fato tem sido apontado como uma ameaça à capacidade da agroecologia de se distinguir da agricultura convencional. Como Costa Neto (2008) problematiza, a construção de uma categoria única de produto reconhecido como 'orgânico' elimina as diferenças entre agroecologia e produção orgânica. Enquanto a primeira estaria associada a uma proposta de revolução agrária, a segunda resume-se a uma técnica de produção orientada para o mercado e sem pretensões transformacionais.

Assim, enquanto do ponto de visa da produção há um debate acerca das distintas denominações para essa busca por modos de produção mais sustentáveis (alternativa, orgânica, biodinâmica, ecológica e, numa concepção mais consolidada do ponto de vista da ciência, agroecológica), do ponto de vista do consumo, o produto dessa produção tem sido reportado de uma categoria genérica 'orgânico' (ALTIERI, 2018; MIGLIORINI e WEZEL, 2017). Isso é, em parte, explicado pela própria legislação, que não estabelece diferenciações na classificação dos produtos oriundos dos distintos modelos de agricultura orgânica. Tanto produtos produzidos dentro dos preceitos da agroecologia quanto um produto oriundo de uma produção em larga escala certificado por auditoria formam uma única categoria de consumo.

A constituição dessa categoria de consumo denominada 'alimentos orgânicos' ocorre por meio de distinções simbólicas em relação aos 'alimentos convencionais', visto que envolvem, além de diferenças na forma de produção, diferenças culturais e mercadológicas (THOMPSON e BALLI, 2007; PRESS et al., 2014). A incorporação de significados de sustentabilidade e saudabilidade aos alimentos orgânicos permite aos orgânicos ocupar uma posição particular no universo cultural dos consumidores (THOMPSON e BALLI, 2007; DALMORO, 2015). Adicionalmente, esses produtos ganham representatividade enquanto uma categoria de consumo quando supermercados e outros canais de comercialização de alimentos passam a adotar práticas mercadológicas distintas, como, por exemplo, utilizando espaços de comercialização específicos para os 'produtos orgânicos' (LOCKIE et al., 2002). 
Com isso, os autores entendem que o futuro dos orgânicos é dependente da motivação dos consumidores em se tornarem 'consumidores de orgânicos'.

Nesse sentido, tão importante quanto as discussões acerca das diferentes concepções de 'orgânico', está o reconhecimento desses produtos como uma categoria de consumo particular em relação aos alimentos em geral (THOMPSON e BALLI, 2007). Isso também passa por padrões de consumo e perfis de consumidores igualmente distintos em relação àqueles encontrados nos alimentos tradicionais. Caso contrário, o caráter 'orgânico' pode ser reduzido a apenas um atributo de um produto. Dessa forma, emerge a necessidade de compreender o perfil dos consumidores de orgânicos, não só como um suporte mercadológico, mas também, de reflexão sobre a própria dinâmica de estabilização de uma categoria de consumo 'orgânico' a despeito das diferentes concepções que envolvem a produção desse tipo de produto.

Esforços teóricos para mapear o perfil de consumo de orgânicos

Diante da eminência do papel do consumidor na configuração da categoria de consumo 'orgânicos', desde o final da década de 1980 pesquisadores têm buscado mapear as particularidades no consumo desses alimentos e características singulares dos consumidores (JOLLY et al., 1989). Estudos que remetem àquele período apontam que, no contexto americano, os consumidores encontraram nos alimentos orgânicos uma alternativa para expressar suas preocupações com o meio-ambiente (DAVIES et al., 1995). Esses pesquisadores relatavam com surpresa o fato de os consumidores americanos pesquisados não reportarem motivações de saúde de maneira clara no consumo de orgânicos. A explicação para isso pode ser encontrada no momento e no contexto em que o estudo foi realizado, pois, no início da década de 1990, havia um clamor internacional em torno das questões ambientais, estimulando formas de consumo reconhecidas como mais éticas e ambientalmente orientadas (FOTOPOULOS e KRYSTALLIS, 2002).

Por outro lado, no contexto europeu, uma série de escândalos alimentares, como a contaminação de alimentos pela bactéria salmonela, epidemias em rebanhos bovinos, preocupação com a segurança de novas técnicas de cozimento, reduziram a confiança dos europeus nos alimentos manufaturados e os orgânicos in natura passaram a ser reconhecidos como produtos mais confiáveis (DAVIES et al., 1995). Assim, o consumo de alimentos orgânicos na Europa também está associado a uma preocupação com a saúde (ZAKOWSKA-BIEMANS, 2011).

Com base nessas variações das razões e perfis no consumo de orgânicos no mundo, diferentes abordagens têm buscado compreender o fenômeno. Uma das abordagens recentes aborda a construção de significados e práticas culturais envolvidos no consumo de alimentos orgânicos (THOMPSON e COSKUNER-BALLI, 2007; DALMORO, 2015; NUTTAVUTHISIT e TH ØGERSEN, 2017). Essa perspectiva prevê que as relações de consumo em torno desse produto não se limitam à lógica econômica ou a fatores psicológicos, mas são orientadas principalmente por construções culturais. Como exemplo, nessa linha, Dalmoro (2015), analisando o contexto brasileiro, descreve que os consumidores constroem em torno dos alimentos orgânicos significados de saudabilidade. Já Thompson e Coskuner-Balli (2007) descrevem um processo de cooptação dos significados particulares dos alimentos orgânicos pelo agronegócio convencional, desestabilizando e alterando a ordem simbólica que os consumidores têm sobre esse tipo de alimento.

No entanto, os estudos que seguem essa linha cultural não dão conta de descrever aspectos individuais do comportamento do consumidor. Assim, outro leque de estudos busca compreender as razões de compra, principalmente buscando trazer comparativos e generalizações no mapeamento do comportamento psicossocial dos consumidores, um exemplo vem de Thøgersen et al. (2015) os quais afirmam que brasileiros, chineses, europeus e americanos possuem valores pessoais similares frente aos orgânicos. Essa conclusão é corroborada por outros estudos que encontraram resultados similares em outros contextos, reforçando que consumidores de orgânicos compartilhariam de valores como segurança, hedonismo, universalismo, benevolência, autocontrole e conformidade (AERTSENS et al. 
2009). Nessa linha, os aspectos psicossociais que ditam o comportamento do consumidor de alimentos orgânicos seriam aspectos transculturais, com pouca variação entre consumidores ao redor do mundo.

A contribuição desse tipo de estudo reside, principalmente, no suporte a uma expansão global dos alimentos orgânicos, tendo impacto dentro da gestão de marketing e da própria popularização do consumo desses alimentos. Por outro lado, ao mesmo tempo em que não estima esforços de valorização e distinção local dos orgânicos - especialmente dentro de uma concepção agroecológica (ALTIERI, 2018), esse tipo de estudo não dá conta de descrever as variações nos perfis de consumo em diferentes contextos.

A compreensão do perfil de consumo dos alimentos orgânicos tem sido buscada, sobretudo, por levantamentos longitudinais, como, por exemplo, a "Hartman Group's Surveys", conduzida regularmente no mercado americano (HARTMAN GROUP, 2006). Enquanto no seu início o levantamento focava em aspectos de comercialização, desde 2004 ele tem focado no entendimento do perfil de consumo e aspectos sociodemográficos dos consumidores. Outro exemplo de estudo longitudinal é o Barômetro dos Orgânicos, conduzido pela Junta Comercial da região da Galícia, na Espanha (CRAEGA, 2018).

Enquanto estudos do ponto de vista cultural e psicossocial apresentam uma expectativa de contribuição especialmente teórica, a compreensão do perfil de consumo demostra potencial imediato no desenvolvimento de ações práticas em prol desse tipo de produto (DIMITRI e DETTMANN, 2011; ANDRADE e BERTOLDI, 2012). Contudo, os resultados das pesquisas realizadas ao redor do mundo têm se caracterizado por inúmeras contradições em relação ao perfil de consumo de orgânicos. Essa variação ocorre, essencialmente, em relação às características sociais e demográficas dos consumidores, bem como suas percepções e motivações de consumo (DIMITRI e DETTMANN; GRUBOR e DJOKIC, 2016). O Quadro 01 apresenta uma lista de estudos realizados em diferentes países e os seus principais achados em termos de perfil de consumo de orgânicos.

Quadro 1. Perfil dos consumidores de orgânicos em diferentes países

\begin{tabular}{|c|c|c|}
\hline Estudo & $\begin{array}{l}\text { Local do } \\
\text { Estudo }\end{array}$ & Principais achados \\
\hline $\begin{array}{ll}\text { Fotopoulos } & \text { e } \\
\text { Krystallis (2002) } & \end{array}$ & Grécia & $\begin{array}{l}\text { Consumidores de orgânicos têm maior renda e escolaridade mais elevada do } \\
\text { que aqueles que não consomem. São predominantemente mulheres, na faixa de } \\
20 \text { a } 50 \text { anos. O estudo identifica, ainda, três tipos de atitudes frente aos } \\
\text { orgânicos: os despreocupados, os preocupados, mas não consumidores, e os } \\
\text { compradores. }\end{array}$ \\
\hline Davies et al. (1995) & $\begin{array}{l}\text { Irlanda } \\
\text { Norte }\end{array}$ & $\begin{array}{l}\text { Consumidores de orgânicos são predominantemente mulheres na faixa de } 30 \text { a } \\
45 \text { anos e que possuem filho. Também possuem escolaridade e renda mais } \\
\text { elevada. O principal motivo para compra é saúde, seguido de questões } \\
\text { ambientais. }\end{array}$ \\
\hline $\begin{array}{l}\text { Dettmann e Dimitri, } \\
2009\end{array}$ & $\begin{array}{l}\text { Estados } \\
\text { Unidos }\end{array}$ & $\begin{array}{l}\text { Consumidores de orgânicos são, predominantemente, pessoas com origens africanas } \\
\text { e asiáticas. Escolaridade e renda mais elevadas também são preditores do consumo } \\
\text { de orgânicos. }\end{array}$ \\
\hline $\begin{array}{l}\text { Hamzaoui-Essoussi } \\
\text { e Zahaf (2012) }\end{array}$ & Canadá & $\begin{array}{l}\text { Consumidores de orgânicos são predominantemente mulheres, solteiras, } \\
\text { profissionalmente ativas e na faixa de } 25 \text { a } 35 \text { anos. Na média, possuem renda } \\
\text { elevada e residem em áreas urbanas. Contudo, os resultados mais significativos } \\
\text { residem nos estilos de vida e não nos aspectos demográficos. Os estilos de vida } \\
\text { também são indicativos do desejo do consumidor em pagar um preço superior aos } \\
\text { orgânicos. }\end{array}$ \\
\hline $\begin{array}{l}\text { Grubor e } \text { Djokic } \\
(2016)\end{array}$ & Sérvia & $\begin{array}{l}\text { Consumidores de orgânicos são predominantemente mulheres, } \\
\text { profissionalmente ativas, casadas e com filhos. Os consumidores valorizam } \\
\text { dietas que proporcionam ganhos de saúde e um grande número teve } \\
\text { experiência de doenças com pessoas próximas, acreditando que o consumo de } \\
\text { orgânicos contribui para prevenir essas doenças. }\end{array}$ \\
\hline Lockie et al. (2002) & Austrália & $\begin{array}{l}\text { Consumidores de orgânicos são principalmente mulheres, mas não apresentam } \\
\text { variação em termos de renda e valores políticos e ambientais. Os autores afirmam } \\
\text { que os consumidores não se caracterizam por ter valores ambientais genuínos, mas } \\
\text { sim contradições entre preocupações ambientais, de saúde e de segurança } \\
\text { alimentar. }\end{array}$ \\
\hline Monier et al. (2009) & França & icos não apresentam diferenças em relação à renda, idade, \\
\hline
\end{tabular}




\begin{tabular}{|l|l|l|}
\hline \multicolumn{1}{|l|}{} & & $\begin{array}{l}\text { gênero ou condição da estrutura familiar. A única diferença significativa identificada } \\
\text { diz respeito ao fato de os consumidores de orgânicos apresentarem, em média, uma } \\
\text { renda mais elevada. Contudo, identificou-se que os consumidores são sensíveis ao } \\
\text { preço. }\end{array}$ \\
\hline $\begin{array}{l}\text { Janssen et al. } \\
(2009) .\end{array}$ & Alemanha & $\begin{array}{l}\text { Esse estudo não encontrou diferenças significativas em relação ao perfil } \\
\text { sociodemográfico dos consumidores de orgânicos. O estudo indica, ainda, a } \\
\text { existência de grupos com alta preferência por orgânicos e outros três com que } \\
\text { apresentam baixo consumo, demonstrando que, enquanto os consumidores na } \\
\text { Alemanha possuem um padrão de consumo mais similar, os não-consumidores } \\
\text { formam um grupo heterogêneo. }\end{array}$ \\
\hline $\begin{array}{l}\text { Roitner- } \\
\text { Schobesberger et al. } \\
(2008)\end{array}$ & Tailândia & $\begin{array}{l}\text { Os consumidores tendem a ser mais velhos, com alta renda familiar. A principal razão } \\
\text { para a compra é o ganho de saúde e a principal barreira é a dificuldade em } \\
\text { diferenciar orgânicos e convencionais. }\end{array}$ \\
\hline
\end{tabular}

Contudo, apesar desses estudos fazerem alusão à existência de um perfil de consumidores distinto por país, as variações podem ocorrer inclusive dentro do próprio país. Isso é especialmente relevante em países com realidades sociodemográficas distintas em cada região, como o caso brasileiro. Como pode ser observado no Quadro 2, estudos realizados em diferentes cidades do Brasil apresentam variações no perfil dos consumidores.

Quadro 2. Perfil do consumidor de alimentos orgânicos no Brasil

\begin{tabular}{|c|c|c|}
\hline Estudo & Local do Estudo & Principais achados \\
\hline Moraes et al. (2015) & $\begin{array}{l}\text { Campo Grande, } \\
\text { MS. }\end{array}$ & $\begin{array}{l}\text { Consumidores de orgânicos são, predominantemente, do sexo feminino, possuem } \\
\text { mais de } 40 \text { anos, são casadas, a maioria possui curso superior, com renda entre } 6 \text { e } \\
10 \text { salários mínimos. O estudo afirma que, embora os consumidores demonstrem } \\
\text { conhecimento sobre os orgânicos, o consumo ainda é restrito a uma pequena parte } \\
\text { da população. }\end{array}$ \\
\hline $\begin{array}{l}\text { Lima-Filho e Silva } \\
\text { (2012) }\end{array}$ & $\begin{array}{l}\text { Campo Grande, } \\
\text { MS. }\end{array}$ & $\begin{array}{l}\text { Os consumidores de orgânicos possuem renda e escolaridade superior. O } \\
\text { consumo é motivado por serem mais saborosos, mais seguros quanto à saúde } \\
\text { e higiene, bem como pela aparência. }\end{array}$ \\
\hline $\begin{array}{l}\text { Andrade e Bertoldi } \\
(2012)\end{array}$ & $\begin{array}{l}\text { Belo } \\
\text { Horizonte, MG. }\end{array}$ & $\begin{array}{l}\text { Consumidores de orgânicos são, predominantemente, mulheres. Com idade } \\
\text { superior a } 30 \text { anos, união estável, ensino superior completo e renda familiar } \\
\text { elevada. A motivação de consumo relaciona-se aos beneficios à saúde. }\end{array}$ \\
\hline $\begin{array}{l}\text { Sampaio e Gosling } \\
\text { (2014) }\end{array}$ & $\begin{array}{l}\text { Belo } \\
\text { Horizonte, MG. }\end{array}$ & $\begin{array}{l}\text { Consumidores de orgânicos são, predominantemente, mulheres, } 46 \text { a } 55 \text { anos, } \\
\text { superior completo, casadas, sem filhos e renda familiar acima de } \mathrm{R} \$ 10 \text { mil. Os } \\
\text { consumidores manifestam uma crença no respeito e preservação do meio- } \\
\text { ambiente e na busca por uma melhoria de saúde. }\end{array}$ \\
\hline $\begin{array}{l}\text { Pereira et } \begin{array}{l}\text { al. } \\
(2015)\end{array} \\
\end{array}$ & Pelotas, RS. & $\begin{array}{l}\text { Consumidores de orgânicos são mulheres, com uma idade média de } 49 \text { anos, renda } \\
\text { de } 2 \text { a } 5 \text { salários mínimos e menor nível de escolaridade. Esses consumidores } \\
\text { manifestam preocupação com a saúde e uma alimentação mais saudável, sem } \\
\text { necessariamente apresentar diagnóstico de doenças que estimulem tal } \\
\text { comportamento. }\end{array}$ \\
\hline $\begin{array}{l}\text { Santos e } \\
\text { Silva Júnior (2015) }\end{array}$ & $\begin{array}{l}\text { Agreste de } \\
\text { Pernambuco. }\end{array}$ & $\begin{array}{l}\text { Consumidores de orgânicos são predominantemente homens, com uma faixa } \\
\text { etária, escolaridade e renda mais elevada. Esse estudo também descreve a } \\
\text { particularidade de serem viúvos. Mesmo sem apresentar as características do } \\
\text { consumo, o estudo chama a atenção pela particularidade no perfil dos } \\
\text { consumidores em relação a outros estudos. }\end{array}$ \\
\hline $\begin{array}{lll}\text { Hoppe } & \text { et } & \text { al. } \\
(2013) & & \end{array}$ & $\begin{array}{l}\text { Porto Alegre, } \\
\text { RS. }\end{array}$ & $\begin{array}{l}\text { Consumidores de orgânicos são predominantemente mulheres, com ensino médio } \\
\text { completo, filhos e idade entre } 31 \text { a } 65 \text { anos. Os motivadores relacionam-se ao fato } \\
\text { de os orgânicos serem mais saudáveis, saborosos, naturais, ambientalmente } \\
\text { corretos, apesar de serem visualmente menos atraentes e mais caros. }\end{array}$ \\
\hline
\end{tabular}

A explicação para essas variações reside, em parte, no fato de que cada estudo adota uma metodologia distinta. A escolha dos produtos pesquisados, a escolha das amostras e das técnicas de coleta de dados pode explicar a variação dos resultados (GRUBOR e DJOKIC, 2016). No entanto, deve-se considerar, também, que a variação é decorrente do fato de que cada região possui particularidades de renda, estilo de vida, produtos disponíveis, entre outras variáveis macrossociais e culturais que podem condicionar o acesso e o tipo de consumo de alimentos (HUGHNER et al., 2007) Consequentemente, a descrição do perfil de consumo de alimentos orgânicos passa pela identificação de diferentes variáveis 
capazes de condicionar estas variações e sua influência na formação do perfil dos consumidores (DETTMANN e DIMITRI, 2009; HUGHNER et al., 2007; SULTAN et al., 2018). Assim, apesar do amplo esforço teórico e gerencial, a descrição do perfil de consumo de orgânicos consiste numa atividade constantemente relevante, visando dar conta das mudanças ao longo do tempo e das particularidades locais e regionais.

\section{Método da pesquisa}

No desenvolvimento do plano empírico, o estudo assumiu um caráter descritivo por meio de uma abordagem quantitativa. Esse tipo de abordagem parte do pressuposto de que a realidade social pode ser mensurada de forma objetiva, traduzida na forma de números (HAIR et al., 2015). A possibilidade de generalização dos resultados oriundos de pesquisas quantitativas para toda a população estudada justificou seu uso nesse estudo, dado o objetivo de analisar o perfil de consumo de uma determinada população.

Para a coleta de dados, foi conduzido um levantamento (tipo survey) com corte transversal, por meio de questionário fechado, visando obter informações pela interrogação direta com unidades amostrais da população. Para isso, foi feito uso de um questionário, aplicado com base nas etapas sugeridas por Fowler (2013), envolvendo: amostragem, questionário, coleta e análise dos dados.

No processo de amostragem, a população foi definida como todos os habitantes do estado do Rio Grande do Sul. Esse estado se destaca como um dos principais polos consumidores de alimentos orgânicos, visto que a média de consumo é cerca de duas vezes maior que a média nacional (ORGANIS, 2017). Segundo informações do IBGE, a população do Rio Grande do Sul é de 11.322.895, distribuídos dissemelhantemente em 497 municípios (IBGE, 2018). A amostra obtida neste estudo foi de 2.691 respondentes. Isso configura uma amostra significativa com margem de erro de $2 \%$ e um intervalo de confiança de 99\% (FOWLER, 2013). A seleção dos respondentes foi não probabilística por conveniência (HAIR et al., 2015), pois foram escolhidos pela sua disponibilidade de participação na pesquisa. Os respondentes residiam em 78 municípios do Estado do RS, sendo a maioria deles na região do Vale do Rio dos Sinos e Região Metropolitana-Delta do Jacuí.

O questionário foi construído pelos pesquisadores a partir do instrumento "Barômetro de percepção e consumo de Alimentos Ecológicos" (CRAEGA, 2018). Esse instrumento foi desenvolvido e testado para mapear de forma longitudinal o perfil dos consumidores de orgânicos na Espanha. Apesar de consistir num instrumento validado previamente, ainda não havia sido utilizado na operacionalização de estudos científicos. Para adequar o instrumento aos propósitos deste estudo, foram incluídas questões complementares, bem como as questões extraídas do questionário original foram traduzidas e adaptadas para o contexto do Rio Grande do Sul. O novo questionário foi inicialmente revisado por um grupo de especialistas em pesquisas de comportamento do consumidor e consumo de orgânicos e prétestado junto a 10 consumidores. A versão final do questionário continha quatro seções: comportamento da compra, percepção acerca dos orgânicos, aspectos mercadológicos e perfil sociodemográfico dos entrevistados.

A coleta de dados ocorreu no segundo semestre de 2016. Visando obter uma cobertura geográfica de diferentes regiões do estado, os questionários foram aplicados presencialmente por um grupo de pesquisadores previamente instruídos pelos coordenadores da pesquisa. Os pesquisadores se dirigiam até diferentes espaços públicos (universidades, supermercados, feiras, residências, entre outros) e coletaram as respostas diretamente com os respondentes, em questionários impressos. Posteriormente, esses questionários foram tabulados em uma planilha eletrônica para a análise dos dados. Para tal, foram adotados testes de estatística descritiva e de variância de média, contanto com o auxílio dos softwares IBM SPSS Statistics 23 e Microsoft Excel. 
Análise dos resultados

\section{Perfil Sócio-demográfico dos Respondentes}

Os dados obtidos foram analisados à luz do objetivo do estudo, visando, primeiramente, compreender o perfil dos respondentes para depois identificar as características do consumo de orgânicos. Diante disso, o perfil demográfico dos entrevistados revela que $57,0 \%$ corresponde ao gênero feminino, 41,8\% masculino e 1,2\% não respondeu, com idade média de 27,7 anos. Em relação ao estado civil, verificou-se que $54,4 \%$ são solteiros, $36,9 \%$ casados, 3,5\% divorciados, $2,7 \%$ informaram que são separados, viúvos ou outro e $2,5 \%$ não informaram. Além disso, a maior parte dos entrevistados informou não possuir filhos (64,2\%), 18,1\% possui 1 filho, 11,4\% 2 filhos, 3,3\% 3 filhos, 1,7\% 4 ou mais filhos, e 1,3\% dos entrevistados não respondeu ao questionamento.

No que diz respeito ao grau de escolaridade dos entrevistados, observou-se que a maior parte dos entrevistados possui um grau de instrução mais elevado, sendo que $43,7 \%$ ensino superior incompleto, $14,5 \%$ ensino superior completo e 10,3\% são pós-graduados. Em contrapartida, apenas 2,8\% possuem ensino fundamental incompleto e $2,7 \%$ ensino fundamental completo.

A maior parte dos entrevistados possui um nível de renda familiar elevado, o que pode estar diretamente relacionado ao nível de instrução dos respondentes. Do total de entrevistados, $28,6 \%$

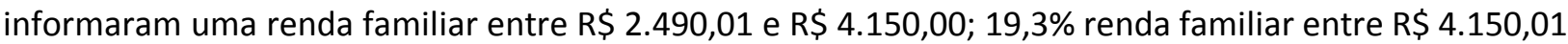
e $\mathrm{R} \$ 6.225,00 ; 10,2 \%$ apresentaram uma renda familiar entre $\mathrm{R} \$ 6.225,01$ e $\mathrm{R} \$ 10.375,00$ e, ainda, $6,5 \%$ possuem renda familiar acima desse valor. Por outro lado, $35,4 \%$ dos entrevistados responderam que a renda familiar é inferior a $\mathrm{R} \$ \mathbf{2 . 4 9 0 , 0 0}$ ou não informaram nenhum valor.

Além disso, os entrevistados também informaram que os núcleos familiares dependentes dessa renda são relativamente pequenos (em média 2,8 pessoas). Quando questionados sobre o número de pessoas que dependem da renda familiar, $28,3 \%$ responderam que três pessoas usufruem dessa renda, 24,5\% informaram que duas pessoas dependem da renda familiar e 13,3\% relataram que uma pessoa é dependente da renda. Em contrapartida, apenas $6,6 \%$ informaram que cinco ou mais pessoas são dependentes da renda familiar. Além disso, $8 \%$ dos entrevistados não responderam ao questionamento. Esses resultados referentes ao perfil amostral se assemelham em relação à distribuição de gênero representada em amostras de outros estudos realizados no Rio Grande do Sul (HOPPE et al., 2013), contudo se diferenciam em termos de idade, nível educacional e renda. Esse fato se deve, principalmente, em função do estudo de Hoppe et al. (2013) focar em estudos da região metropolitana, enquanto que os dados deste estudo incluem consumidores do interior do estado. Além disso, os resultados reforçam as variações amostrais entre diferentes estudos, ressaltando a importância da condução constante de novos estudos sobre o tema.

\section{Comportamento de Compra de Orgânicos}

No que se refere ao comportamento de compra, os respondentes manifestaram um comportamento variável quando questionados sobre há quanto tempo consomem ou não alimentos orgânicos. Ao mesmo tempo em que $24,9 \%$ dos entrevistados não consomem alimentos orgânicos e $20,5 \%$ consomem há apenas um ano, 28,5\% da amostra consomem orgânicos há mais de 5 anos. Ademais, $20,5 \%$ da amostra relatou que mudou seus hábitos de consumo de alimentos orgânicos durante o último ano.

Ao serem questionados sobre as compras semanais de alimentos, os entrevistados responderam que, em média, $22,28 \%$ dos produtos adquiridos caracterizam-se como orgânicos (com desvio padrão igual a 21,32 ), sendo o maior consumo de vegetais e hortaliças $(68,4 \%)$, frutas $(66,3 \%)$ e ovos (39,2\%), conforme Tabela 1, que apresenta a relação dos produtos orgânicos que os entrevistados costumam consumir. Por outro lado, $14,5 \%$ dos respondentes informaram não ter o hábito de consumir esse tipo de produto. 


\begin{tabular}{lcc} 
Tabela 1. Produtos orgânicos consumidos & & \\
\hline Produto* & $\mathbf{N}$ & \% \\
\hline Vegetais e hortaliças & 1.840 & 68,4 \\
Frutas & 1.785 & 66,3 \\
Ovos & 1.055 & 39,2 \\
Leite e derivados & 565 & 21,0 \\
Carne & 529 & 19,7 \\
Cereais & 394 & 14,6 \\
Bebidas & 280 & 10,4 \\
Doces & 230 & 8,5 \\
Conservas, pratos prontos ou similares & 205 & 7,6 \\
Outros & 21 & 0,8 \\
Não tem o hábito de consumir & 391 & 14,5 \\
\hline
\end{tabular}

*Nesta questão era possível marcar mais de uma alternativa

Em relação aos critérios analisados no momento da compra de um alimento, $81,1 \%$ dos respondentes destacou a sua qualidade, $59,9 \%$ respondeu que avaliam o seu preço e $48,2 \%$ avaliam o frescor dos alimentos (Tabela 2). Comparativamente, esses também foram os critérios mais citados em pesquisa realizada na Espanha (CRAEGA, 2018). Em contrapartida, entre os critérios menos destacados estão a sua certificação de qualidade $(13,8 \%)$, o fato de estes prejudicarem o meio ambiente $(11,3 \%)$, ou, ainda, estarem associados a outros fatores $(1,3 \%)$.

Tabela 2. Critérios analisados no momento da compra

\begin{tabular}{lcc}
\hline Critérios* & $\mathbf{N}$ & $\mathbf{\%}$ \\
\hline Sua qualidade & 2.217 & 82,4 \\
Seu preço & 1.637 & 60,8 \\
O frescor dos alimentos & 1.296 & 48,2 \\
Se eles não têm agrotóxicos & 689 & 25,6 \\
Sua origem & 630 & 23,4 \\
Sua marca & 609 & 22,6 \\
O estabelecimento & 495 & 18,4 \\
Se possuem certificação de qualidade & 372 & 13,8 \\
Se eles prejudicam o meio ambiente & 303 & 11,3 \\
Outro & 34 & 1,3 \\
\hline
\end{tabular}

*Nesta questão era possível marcar mais de uma alternativa

No que diz respeito aos motivos associados ao início do consumo de alimentos orgânicos ou a sua continuidade, os participantes da amostra declaram como principal a saúde $(69,9 \%)$, seguida pela opção de consumir produtos produzidos sem pesticidas ou fertilizantes químicos $(55,1 \%)$ e para ter uma alimentação sem corantes ou conservantes químicos (52,8\%) (Tabela 3). Esses resultados corroboram informações divulgadas em outros estudos (VILAS BOAS et al., 2006; HASIMU et al., 2017). Além disso, entre os outros motivos que também foram destacados, porém com menor importância, estão: sua maior qualidade, sua preocupação em cuidar da natureza, seu sabor, sua relação direta com o campo, entre outros.

Os entrevistados também responderam um conjunto de questões associadas às suas percepções acerca dos orgânicos. Neste sentido, no que se refere às características associadas aos alimentos orgânicos, $71,6 \%$ dos entrevistados percebem os alimentos orgânicos como naturais, $56,8 \%$ da amostra também entendem que os orgânicos são alimentos livres de transgênicos, 55,2\% enxergam os orgânicos como alimentos saudáveis, sem aditivos químicos e $54,6 \%$ veem os orgânicos como aqueles produzidos sem pesticidas químicos (Tabela 4).

Por outro lado, os entrevistados destacaram que o consumo de orgânicos: contribui para evitar riscos como doenças ou agressão ao organismo, associadas aos agrotóxicos para 57,2\%; garante a qualidade do produto na percepção de $44,4 \%$ da amostra; estimula uma alimentação melhor, segundo $39,4 \%$ dos entrevistados, entre outras variáveis apresentadas na Tabela 5. Entre as consequências associadas ao consumo de orgânicos menos destacadas na pesquisa estão a possibilidade de economia $(2,8 \%)$, o estabelecimento de um relacionamento de confiança com outros $(2,1 \%)$, entre outros $(2,7 \%)$. 
Tabela 3. Motivos para começar ou continuar consumindo alimentos orgânicos

\begin{tabular}{lcc}
\hline Motivos* & N & \% \\
\hline Por questão de saúde & 1.880 & 69,9 \\
Para evitar produtos produzidos com pesticidas ou fertilizantes químicos & 1.484 & 55,1 \\
Para ter uma alimentação sem corantes e conservantes químicos & 1.422 & 52,8 \\
Pela sua qualidade superior & 996 & 37,0 \\
Para cuidar da natureza & 950 & 35,3 \\
Por seu sabor & 887 & 33,0 \\
Por virem diretamente do campo & 488 & 18,1 \\
Para ter a garantia de que os animais foram criados com produtos naturais & 330 & 12,3 \\
Por recomendação & 216 & 8,0 \\
Apenas para provar & 64 & 14 \\
Outros & 2,4 \\
\hline
\end{tabular}

*Nesta questão era possível marcar mais de uma alternativa

No que tange aos valores associados aos alimentos orgânicos, o principal item destacado foi relacionado à qualidade de vida, para $70,3 \%$ dos entrevistados, seguido pela possibilidade de viver bem a vida $(44,9 \%)$ e pela longevidade $(44,2 \%)$ (Tabela 6$)$. Além disso, outros valores destacados ainda com menor importância foram a responsabilidade social $(28,8 \%)$, a tranquilidade $(20,5 \%)$, a felicidade $(20,2 \%)$, o cuidado com os entes queridos $(19,4 \%)$, a harmonia $(19,2 \%)$, a socialização $(4,0 \%)$, entre outros $(1,2 \%)$.

Tabela 4. Características associadas aos alimentos orgânicos

\begin{tabular}{|c|c|c|}
\hline Variável* & $\mathbf{N}$ & $\%$ \\
\hline Naturais & 1927 & 71,6 \\
\hline Livres de Transgênicos & 1528 & 56,8 \\
\hline Saudáveis, sem aditivos ou corantes & 1485 & 55,2 \\
\hline Produzidos sem pesticidas químicos & 1470 & 54,6 \\
\hline Produzidos respeitando o meio ambiente & 904 & 33,6 \\
\hline Mais caros & 904 & 33,6 \\
\hline Artesanais, produzidos tradicionalmente & 565 & 21,0 \\
\hline Cultivo tradicional & 565 & 21,0 \\
\hline Certificados através de controles que garantem seu método de obtenção & 529 & 19,7 \\
\hline Difíceis de encontrar & 513 & 19,1 \\
\hline Integrais & 423 & 15,7 \\
\hline Alimentos transgênicos & 47 & 1,7 \\
\hline De aspecto ruim ou pouca validade & 33 & 1,2 \\
\hline Alimentos dietéticos & 30 & 1,1 \\
\hline Outros & 9 & 0,3 \\
\hline
\end{tabular}

*Nesta questão era possível marcar mais de uma alternativa

Os entrevistados também foram questionados sobre os atributos que associam aos alimentos orgânicos, sendo que o mais destacado por $56,4 \%$ da amostra foi a ausência de agrotóxicos (Tabela 7). Também foram destacados o fato de a produção ocorrer de forma consciente, para $51,6 \%$, e ser mais saudável em relação aos demais alimentos, segundo $42,3 \%$ da amostra. Por outro lado, os atributos menos destacados foram a variabilidade de seu tamanho, a durabilidade e a marca do produto, sendo estes relevantes para $8,9 \%, 4,0 \%$ e $2,7 \%$ dos respondentes, respectivamente. 
Tabela 5. Consequências associadas ao consumo de orgânicos

\begin{tabular}{lcc}
\hline Variável & N & $\%$ \\
\hline Evitar riscos associados aos agrotóxicos & 1540 & 57,2 \\
Garantia da qualidade do produto & 1195 & 44,4 \\
Estímulo a uma alimentação melhor & 1061 & 39,4 \\
Sentir o sabor real & 1037 & 38,5 \\
Sentir segurança ao se alimentar & 1000 & 37,2 \\
Sentir que estou cuidando melhor da saúde da minha família/preocupação com os filhos & 31,4 \\
Confiança na procedência & 846 \\
Preservar a natureza & 801 \\
Sentimento de melhor cuidado com saúde pessoal /autoestima & 29,8 \\
Prazer ao alimentar & 778 \\
Sentir-se socialmente responsável & 722 \\
Valorizar as origens & 28,9 \\
Reduzir despesas e inconvenientes com tratamentos de saúde & 26,8 \\
Armazenar por mais tempo & 396 \\
Sentir-se apto às tarefas diárias e a enfrentar os problemas do dia-a-dia & 23,7 \\
Ter economia & 14,7 \\
Estabelecer relacionamento de confiança com outros & 354 \\
Outros & 13,2 & 288 \\
\hline Nesta questa & 128 & 4,8 \\
\hline
\end{tabular}

*Nesta questão era possível marcar mais de uma alternativa

Tabela 6. Valores associados aos alimentos orgânicos

\begin{tabular}{lcc}
\hline Variável* & N & \% \\
\hline Qualidade de vida & 1893 & 70,3 \\
Viver bem a vida & 1207 & 44,9 \\
Longevidade & 1190 & 44,2 \\
Responsabilidade social & 776 & 28,8 \\
Tranquilidade & 553 & 20,5 \\
Felicidade/alegria de viver & 544 & 20,2 \\
Cuidar dos entes queridos & 523 & 19,4 \\
Harmonia/equilíbrio & 516 & 19,2 \\
Socialização & 108 \\
Outros & 33 & 4,0 \\
\hline
\end{tabular}

*Nesta questão era possível marcar mais de uma alternativa

Quando questionados sobre as palavras que transmitem a imagem positiva para os alimentos orgânicos, as respostas foram similares às anteriormente manifestadas nas características e atributos associados aos orgânicos, de modo que as principais palavras mencionadas foram: saudáveis, natural e livre de agrotóxicos pela maioria dos respondentes, conforme pode ser visualizado na Tabela 8.

A pesquisa buscou identificar, ainda, a compreensão que os respondentes têm acerca da concepção dos alimentos orgânicos. As afirmações mais destacadas se referem a alimentos de origem vegetal (45,6\% dos entrevistados) (Tabela 8 ). Na sequência, as afirmações mais destacadas foram de que alimentos orgânicos são submetidos a inspeções controladas para a sua certificação por $39,8 \%$, e de que apenas produtos frescos e próprios da época podem ser caracterizados como alimentos orgânicos para $33,8 \%$ da amostra. Essas afirmações mencionadas indicam que ainda faltam informações a respeito dos alimentos orgânicos para um grupo significativo da população, mesmo para aqueles com maior nível de escolaridade e renda, sendo necessário, por exemplo, o incentivo de iniciativas de políticas públicas e ações educativas. 
Tabela 7. Atributos associados aos alimentos orgânicos

\begin{tabular}{lcc}
\hline Variável* & N & $\%$ \\
\hline Ausência de agrotóxicos & 1.519 & 56,4 \\
Produzido de forma consciente & 1.388 & 51,6 \\
Mais saudável & 1.137 & 42,3 \\
Melhor sabor & 962 & 35,7 \\
Presença selo/certificação orgânica & 940 & 34,9 \\
Garantia de procedência do produto & 918 & 34,1 \\
Preço alto & 666 & 24,7 \\
Aparência visual natural & 597 & 22,2 \\
Informação na embalagem & 401 \\
O preço do produto & 345 \\
Diversos tamanhos & 239 \\
Durabilidade & 107,9 \\
Marca do produto & 72 & 12,8 \\
\hline
\end{tabular}

* Nesta questão era possível marcar mais de uma alternativa

Tabela 8. Palavras/expressões que transmitem imagem positiva para alimentos orgânicos

\begin{tabular}{lcc}
\hline Palavras/Expressões* & N & $\%$ \\
\hline Saudáveis & 1831 & 68,0 \\
Natural & 1744 & 64,8 \\
Livre de Agrotóxicos & 1700 & 63,2 \\
Do Campo & 910 & 33,8 \\
Ecológico & 769 & 28,6 \\
Caseiro & 754 & 28,0 \\
Artesanal & 386 & 14,3 \\
Regional & 151 & 5,6 \\
Biológico & 149 \\
Tradicional & 102 \\
Nacional & 85 & 5,5 \\
Calórico & 10 \\
Outros & 3,8 \\
\hline
\end{tabular}

*Nesta questão era possível marcar mais de uma alternativa

Em relação aos aspectos mercadológicos, também é possível observar uma disparidade entre os respondentes. Enquanto $33,4 \%$ afirmaram que os estabelecimentos nas cidades onde residem ofertam alimentos orgânicos em quantidade suficiente, $32,1 \%$ observaram que a quantidade é escassa e $25,5 \%$ não responderam à questão ou informaram que não existem alimentos orgânicos disponíveis.

Por fim, o estudo também buscou identificar a compreensão dos respondentes acerca do que consistem 'alimentos orgânicos'. A primeira delas visava identificar quais afirmações estão associadas aos orgânicos, sendo que as respostas que mais se destacaram: os alimentos orgânicos podem ser de origem vegetal $(45,6 \%)$, alimentos orgânicos como aqueles alimentos que são submetidos a inspeções controladas por certificação $(39,8 \%)$ e orgânicos como produtores caracterizados apenas por serem produtos frescos e próprios da época (33,8\% dos entrevistados). No capítulo seguinte são discutidos esses resultados em diálogo com o referencial teórico. 
Tabela 9. Afirmações relacionadas aos alimentos orgânicos

\begin{tabular}{lcc}
\hline Afirmações & N & $\%$ \\
\hline Podem ser de origem vegetal & 1.227 & 45,6 \\
São submetidos a inspeções controladas para sua certificação & 1.070 & 39,8 \\
Apenas produtos frescos e próprios da época podem ser caracterizados como alimentos orgânicos & 910 & 33,8 \\
São produzidos artesanalmente & 789 & 29,3 \\
São produtos orgânicos apenas aquelas que possuem a certificação na embalagem & 627 & 23,3 \\
São o que chamamos de produtos nacionais & 89 & 3,3 \\
\hline
\end{tabular}

\section{Considerações finais}

Tanto estudos acadêmicos quanto de mercado têm fornecido resultados conflitantes em relação ao consumo de alimentos orgânicos (DIMITRI e DETTMANN, 2012; SULTAN et al., 2018). Este estudo contribui com os esforços teóricos no fornecimento de uma análise ampla do perfil e do comportamento de compra de alimentos orgânicos por parte de consumidores gaúchos. Análises realizadas em contextos específicos permitem por luz às particularidades no consumo e no perfil dos consumidores, auxiliando na busca por padrões em relação ao perfil e comportamento do consumo.

Os resultados indicam que, no caso dos consumidores gaúchos, a preocupação com questões associadas à saúde e à preservação ambiental tem sido o principal indutor do consumo de alimentos orgânicos. Os consumidores enxergam, nesse tipo de produto, um alimento mais saudável, natural e livre de agrotóxicos, pesticidas e fertilizantes sintéticos. Os consumidores entendem, ainda, que o consumo de alimentos orgânicos contribui para a redução dos riscos de doenças associadas aos agrotóxicos e garante melhor qualidade de vida.

As principais características sobre o perfil dos consumidores entrevistados podem ser sintetizadas como sendo do gênero feminino, solteiro, com idade média de 27,7 anos, sem filhos, inseridos em núcleos familiares pequenos, cursando o ensino superior e com nível de renda médio elevado. Os principais produtos consumidos pelos entrevistados são vegetais, hortaliças e frutas, sendo os critérios analisados para a sua qualidade, preço e o frescor dos alimentos. A adoção desse tipo de produto nos hábitos alimentares é recente, porém apresenta um crescimento constante, visto que $75,1 \%$ dos entrevistados são consumidores ativos de alimentos orgânicos.

Entre os critérios analisados para a compra de orgânicos foram mencionados sua qualidade, preço e o frescor dos alimentos. Contudo, ao contrário de outros estudos que identificaram o preço como sendo um dos limitadores para o consumo (HAMZAOUI-ESSOUSSI e ZAHAF, 2012; HASIMU et al., 2017), o preço não esteve entre os principais atributos associados aos alimentos orgânicos.

Esses resultados dialogam em parte com estudos prévios, reforçando os aspectos de saudabilidade (ZAKOWSKA-BIEMANS, 2011; DALMORO, 2015) e preocupação ambiental (DAVIES et al., 1995; FOTOPOULOS e KRYSTALLIS, 2002) como os principais indutores ao consumo de alimentos orgânicos. Nesse ponto, diferentemente de contextos como o australiano, a preocupação com a segurança alimentar não ficou tão evidente (LOCKIE et al., 2002). A razão para isso pode estar no fato dos consumidores não reconhecerem um risco alimentar nos alimentos convencionais, mas sim um risco à saúde causado pelos agrotóxicos.

Por outro lado, os resultados reforçam contradições em relação ao perfil dos consumidores comparados a estudos prévios. Mesmo que algumas categorias sociais e demográficas demonstrem um padrão como, por exemplo, o predomínio de mulheres (mantendo um contraste somente em relação ao estudo de Santos e Silva Junior (2015)), questões como estado civil e estrutura familiar demonstram variar de acordo com o estudo. A explicação para isso pode residir nos diferentes processos metodológicos adotados em cada estudo, resultando em características amostrais distintas e que inviabilizam a comparação, bem como nas próprias particularidades de cada contexto. Como argumentado neste artigo, o perfil de consumo de alimentos orgânicos não é homogêneo e universal, mas pode apresentar variações entre diferentes regiões. 
Em relação à contribuição prática, os resultados fornecem dados amplos e capazes de descrever diferentes regiões do estado do Rio Grande do Sul. Esses dados podem ser explorados por setores responsáveis por políticas públicas de estímulo a modos de alimentação mais sustentáveis e saudáveis. A construção de políticas públicas pode se beneficiar mais de variáveis operacionais, descritoras do perfil de consumo, ao invés de reflexões prescritivas de questões psicossociais, visto a capacidade destas retratarem as particularidades do estado do Rio Grande do Sul. Da mesma forma, empresas e produtores rurais que atuam nesse segmento podem encontrar suporte para o desenvolvimento de estratégias adaptadas para este perfil de consumidores. Como Lee e Yun (2015) enfatizam, o crescimento do mercado de alimentos orgânicos está alicerçado na identificação de particularidades no seu consumo, capazes de orientar a busca por vantagens frente aos alimentos convencionais. Estratégias de desenvolvimento do setor no estado do Rio Grande do Sul podem, também, buscar formas de acesso aos $24,1 \%$ da população que ainda não incorporou os alimentos orgânicos nos seus hábitos de consumo.

Com base nessas implicações, ressalta-se que a compreensão do consumo de alimentos orgânicos não é um mero exercício intelectual, mas uma ferramenta à disposição de atores sociais capazes de promoverem o crescimento desse tipo de consumo. Além disso, essa compreensão serve como um alerta na busca por mecanismos de preservação das distinções dessa categoria de bem de consumo em face de sua eminente cooptação de significados (THOMPSON e COSKUNER-BALLI, 2007). Não se trata de sucumbir a uma lógica de segmentação de mercado que reduz os alimentos orgânicos a um mero atributo de compra, mas utilizar a pesquisa como recurso para construção de uma categoria distinta, capaz de reforçar os princípios da agroecologia nas ações de consumo. Esse ponto, inclusive, emerge como uma sugestão de estudos futuros, envolvendo a operacionalização de estudos capazes de identificar as variações no perfil dos consumidores reconhecedores dos princípios da agroecologia em distinção aos consumidores de orgânicos em geral.

Assim, apesar da contribuição gerencial do estudo, os resultados não devem ser lidos apenas como uma ferramenta para incremento da lucratividade do setor, mas como uma forma de estimular o crescimento de um setor capaz de promover ganhos para a sociedade (ANDRADE e BERTOLDI, 2012). Esse entendimento encontra suporte na premissa de que a produção orgânica resulta num menor impacto ambiental quando comparada com a produção convencional. $O$ incremento no consumo de alimentos orgânicos, naturalmente diminui o consumo e a produção de alimentos convencionais (DIMITRI e DETTMANN, 2012). O aumento da demanda por alimentos orgânicos, também, permite a expansão desse tipo de produto para segmentos ainda pouco explorados, tanto em termos de distribuição - visando um acesso mais democrático aos alimentos orgânicos, quanto em termos de variedade de produtos - superando a concentração atual em hortaliças e frutas.

Por fim, é importante ressaltar as limitações do estudo reconhecendo o caráter nãoprobabilístico da amostra. Com isso, os resultados podem sofrer variações em relação ao comportamento da população como um todo. Como forma de superar essas limitações, estimula-se a replicação deste estudo em outros momentos temporais, seguindo a lógica de painéis longitudinais (HARTMAN GROUP, 2006; CRAEGA, 2018). Adicionalmente, diante do argumento teórico de que o perfil de consumo de alimentos orgânicos é ditado por particularidades regionais, estimulam-se novos estudos em outras regiões.

\section{Referências}

AERTSENS, J.; et al. Personal determinants of organic food consumption: a review. British Food Journal, v. 111, s.n., p. 1140-1167, 2009.

ALTIERI, Miguel A. Agroecology: the science of sustainable agriculture. Boca Raton, FL: CRC Press, 2018.

ANDRADE, L. M. S.; BERTOLDI, M. C. Atitudes e motivações em relação ao consumo de alimentos orgânicos em Belo Horizonte-MG. Brazilian Journal of Food Technology, v. 15, n. SSA, p. 31-40, 2012.

BRASIL. Lei no 10.831, de 23 de dezembro de 2003. Disponível em:<http://www.planalto.gov.br/ccivil_03/leis/2003/L10.831.htm.>. Acesso em: 15 de out. 2018.

BURTON, R. J. F. Seeing through the good farmer's eyes: towards developing an understanding of the social symbolic value of productivist behavior. Sociologia Ruralis, v. 44, n. 2, p. 195-215, 2004.

ORGANIS - CONSELHO BRASILEIRO DA PRODUÇÃO ORGÂNICA E SUSTENTÁVEL. Consumo de produtos orgânicos no Brasil: Primeira pesquisa nacional sobre o consumo de orgânicos, 2017. Suplemento. 
CRAEGA - CONSELLO REGULADOR AGRICULTURA ECOLÓXICA DE GALICIA. Percepción e consumo de alimentos ecolóxicos en Galicia. 2o Barómetro. 2013. Disponível em: <https://www.craega.es/a-producionecoloxica/estudos/>. Acesso em: 10 abr. 2018.

COSTA NETO, C. Relações entre agronegócio e agroecologia no Contexto do desenvolvimento rural brasileiro. In FERNANDES, B.M. (org.) Campesinato e agronegócio na América Latina: a questão agrária atual. São Paulo: Expressão Popular, 2008.

DALMORO, M. Construção de significados culturais: uma análise do mercado de suco de uva orgânico. Revista Brasileira de Marketing, v. 14, n. 1, p. 97-109, 2015.

DAVIES, A.; et al. Who buys organic food?: A profile of the purchasers of organic food in Northern Ireland. British Food Journal, v. 97, n. 10, p.17-23, 1995.

DETTMANN, R. L.; DIMITRI, C. Who's buying organic vegetables? demographic characteristics of U.S. consumers. Journal of Food Products Marketing, v. 16, n. 1, p. 79-91, 2009.

DIMITRI, C.; DETTMANN, R. L. Organic food consumers: what do we really know about them?. British Food Journal, v. 114, n. 8, p. 1157-1183, 2012.

FOTOPOULOS, C.; KRYSTALLIS, A. Purchasing motives and profile of the Greek organic consumer: a countrywide survey. British Food Journal, v. 104, n. 9, p. 730-765, 2002.

FOWLER JR, F. J. Survey research methods. London: Sage publications, 2013.

GLOBORURAL. Orgânicos: faturamento do setor deve crescer $20 \%$ este ano. Disponível em: <https://revistagloborural.globo.com/Noticias/Agricultura/noticia/2018/06/globo-rural-organicos-faturamentodo-setor-deve-crescer-20-este-ano-diz-ming-liu.html>. Acesso em: 15 out. 2018.

GRUBOR, A.; DJOKIC, N. Organic food consumer profile in the Republic of Serbia. British Food Journal, v. 118, n. 1, p. 164-182, 2016.

HAIR JR, J. F. et al. Essentials of business research methods. London: Routledge, 2015.

HAMZAOUI-ESSOUSSI, L.; ZAHAF, M. Canadian organic food consumers' profile and their willingness to pay premium prices. Journal of International Food \& Agribusiness Marketing, v. 24, n. 1, p. 1-21, 2012.

HARTMAN GROUP. Organic2006: Consumer attitudes \& behavior five years later \& into the future. Bellevue, WA: Hartman Group, 2006.

HASIMU, H.; et al. A concept mapping study on organic food consumers in Shanghai, China. Appetite, v. 108, s.n., p. 191-202, 2017.

HOPPE, A.; et al. Consumer behaviour towards organic food in Porto Alegre: an application of the theory of planned behaviour. Revista de Economia e Sociologia Rural, v. 51, n. 1, p. 69-90, 2013.

HUGHNER, R. S. ; et al. Who are organic food consumers? A compilation and review of why people purchase organic food. Journal of Consumer Behaviour, v. 6, n. 2/3, p. 94-110. 2007

IBGE - INSTITUTO BRASILEIRO DE GEOGRAFIA E ESTATÍSTICA. Pesquisa Nacional por Amostra de Domicílios

Contínua Trimestre Móvel dezembro 2017 a fevereiro 2018. Disponível em: <ftp://ftp.ibge.gov.br/Trabalho_e_Rendimento/Pesquisa_Nacional_por_Amostra_de_Domicilios_continua/Mens al/Comentarios/pnadc_201802_comentarios.pdf>. Acesso em: 10 abr. 2018.

IFOAM. The IFOAM norms for organic production and processing. Germany: IFOAM-Organics International, 2017

JANSSEN, M.; et al. Is there a promising market in between'organic and conventional food? Analysis of consumer preferences. Renewable Agriculture and Food Systems, v. 24, n. 3, p. 205-213, 2009.

JOLLY, D. A., et al. Organic foods: Consumer attitudes and use. Food Technology, v. 43, n. 11, p. 60-66, 1989.

LEE, H-J.; YUN, Z-S. Consumers' perceptions of organic food attributes and cognitive and affective attitudes as determinants of their purchase intentions toward organic food. Food Quality and Preference, v. 39, n.1, p. 259267, 2015.

LIMA-FILHO, D. O.; SILVA, F. Q. Percepção do consumidor sobre produtos orgânicos. Revista Brasileira de Marketing, v. 11, n. 1, p. 29-46, 2012.

LOCKIE, S.; et al. Eating 'Green': Motivations behind organic food consumption in Australia. Sociologia Ruralis, v. 42, n. 1, p. 23-40, 2002.

MIGLIORINI, P.; WEZEL, A. Converging and diverging principles and practices of organic agriculture regulations and agroecology: a review. Agronomy for Sustainable Development, v. 37, n. 6, p. 63, 2017.

MONIER, S. et al. Organic Food Consumption Patterns. Journal of Agricultural \& Food Industrial Organization, v. 7, n. 2, pp. 1-23, 2009.

MORAES, M. L. et al. Analise do perfil dos consumidores de produtos orgânicos de Campo Grande, Mato Grosso do Sul. Cadernos de Agroecologia, v. 9, n. 4, p. 55-62, 2015.

NUTTAVUTHISIT, K.; TH $\varnothing$ GERSEN, J. The importance of consumer trust for the emergence of a market for green products: the case of organic food. Journal of Business Ethics, v. 140, n. 2, p. 323-337, 2017.

PEREIRA, M. C. et al. Mudança no perfil sociodemográfico de consumidores de produtos orgânicos. Ciencia \& Saúde Coletiva, v. 20, n. 9, p. 2797-2804, 2015. 
PRESS, M.; et al. Ideological challenges to changing strategic orientation in commodity agriculture. Journal of Marketing, v. 78, n. 6, p. 103-119, 2014.

ROITNER-SCHOBESBERGER, B.; et al. Consumer perceptions of organic foods in Bangkok, Thailand. Food Policy, v. 33, n. 2, p. 112-121, 2008.

SAMPAIO, D. O.; GOSLING, M. Consumers of organic food and sustainable development in Brazil, World Journal of Entrepreneurship, Management and Sustainable Development, v. 10, n. 1, p.77-86, 2014.

SANTOS, J. S.; SILVA JÚNIOR, L. H. Determinantes socioeconômicos do consumo e disposição a pagar por alimentos orgânicos no agreste de Pernambuco. Reflexões Econômicas, v. 1, n. 1, p. 49-84, 2015.

SULTAN, P.; et al. Segmenting the Australian organic food consumer market. Asia Pacific Journal of Marketing and Logistics, v. 30, n. 1, p. 163-181, 2018.

SUTHERLAND, L. A.; DARNHOFER, I. Of organic farmers and 'good farmers': changing habitus in rural England. Journal of Rural Studies, v. 28, n. 3, p. 232-240, 2012.

TH $\varnothing$ GERSEN, J.; et al. Consumer buying motives and attitudes towards organic food in two emerging markets: China and Brazil, International Marketing Review, v. 32, n. 3/4, p.389-413, 2015

THOMPSON, C. J.; COSKUNER-BALLI, G. Countervailing market responses to corporate co-optation and the ideological recruitment of consumption communities. Journal of Consumer Research, v. 34, n. 2, p. 135-152, 2007.

VILAS BOAS, L. H. B.; et al. Comportamento do consumidor de produtos orgânicos: uma aplicação da teoria da cadeia de meios e fins. Organizações Rurais \& Agroindustriais, v. 8, n. 1, p.25-39, 2006.

ŻAKOWSKA-BIEMANS, S. Polish consumer food choices and beliefs about organic food. British Food Journal, v. 113, n. 1, p.122-137, 2011. 